\title{
Kondo Effect from a Tunable Bound State within a Quantum Wire
}

\author{
F. Sfigakis, ${ }^{*}$ C. J. B. Ford, M. Pepper, M. Kataoka, D. A. Ritchie, and M. Y. Simmons ${ }^{\dagger}$ \\ Cavendish Laboratory, J. J. Thomson Avenue, Cambridge, CB3 OHE, United Kingdom
}

(Received 30 May 2007; published 17 January 2008)

\begin{abstract}
We investigate the conductance of quantum wires with a variable open quantum dot geometry, displaying an exceptionally strong Kondo effect and most of the 0.7 structure characteristics. Our results indicate that the 0.7 structure is not a manifestation of the singlet Kondo effect. However, specific similarities between our devices and many of the clean quantum wires reported in the literature suggest a weakly bound state is often present in real quantum wires.
\end{abstract}

At low temperatures, the differential conductance $G=$ $d I / d V$ of quantum wires is quantized in units of $2 e^{2} / h$, due to the transmission of spin-degenerate onedimensional (1D) subbands [1,2]. However, a shoulder near the conductance value $\sim 0.7\left(2 e^{2} / h\right)$ can often be observed and is referred to as the " 0.7 anomaly" or " 0.7 structure" [3]. Its origin is still under debate. References [4,5] highlight similarities between the Kondo effect in quantum dots [6-10] and the 0.7 structure. They attribute all observed properties of the 0.7 structure to the suppression (with temperature $T$, magnetic field $B$, or source-drain bias $V_{\text {sd }}$ ) of a 1D Kondo-like enhancement of the conductance above a fully spin-polarized $0.5\left(2 e^{2} / h\right)$ plateau. Many theoretical investigations have predicted the formation of a potential minimum for at least one spin type in quantum wires [11-17].

In this Letter, we report on quantum wires whose geometry favors the formation of a bound state within the 1D channel. These devices, akin to open quantum dots, exhibit an exceptionally strong Kondo effect, as well as all properties associated with the 0.7 structure. However, behavior due to Kondo physics can be distinguished from that due to "0.7 structure physics." We thus propose that both the Kondo-like effect in 1D channels and the 0.7 structure are separate and distinct effects.

Data in this Letter came from the most comprehensively studied device from a set of three devices, all fabricated by etching a $1 \mathrm{D}$ channel in a GaAs/AlGaAs $2 \mathrm{D}$ electron gas with carrier density and mobility of $1.82 \times 10^{15} \mathrm{~m}^{-2}$ and $475 \mathrm{~m}^{2} / \mathrm{V} \mathrm{s}$, respectively, and depositing metal gates into the etched regions. Key characteristics were reproduced before and after illumination, and on several cooldowns from room temperature.

The inset of Fig. 1(a) reveals two protrusions (indicated by arrows) from the etched regions into the $(264 \pm 10) \mathrm{nm}$ wide 1D channel. These form micro-constrictions at the entrance and exit $[(239 \pm 10) \mathrm{nm}$ and $(246 \pm 10) \mathrm{nm}$ wide], thereby creating a shallow bound state inside a double-barrier potential along the length of the wire. As a result, a single resonant peak is present in the riser of the first quantized conductance plateau. This resonant peak disappears in Fig. 1(b) when the conducting channel is moved to either side, away from the center of the etched channel, by differentially biasing the left and right gates by $\Delta V_{g}$ (only $\Delta V_{g}>0$ is shown). With increasing $\left|\Delta V_{g}\right|$, the entrance and exit barrier potentials become increasingly asymmetric, until the resonant peak is destroyed and only one of the two micro-constrictions dominates 1D transport, as in Fig. 1(a).

Figure 2(a) shows that the single resonant peak has no corresponding peak in the risers of higher plateaux. This is not the behavior of length resonances (typically, the amplitude increases with subband index [18-20]), and thus we interpret this resonant peak to be a Coulomb-Blockade (CB) peak. We believe the conductance dip observed most strongly on the first plateau results from resonant backscattering when the second 1D subband opens only in the widening between the micro-constrictions [21]. The behavior in Fig. 2(b) is quite similar to the singlet Kondo effect seen in quantum dots [6-10]. Figure 2(c) shows that $G$ increases with increasing $T$ : the Kondo effect is completely suppressed. An additional shoulder (indicated by an arrow) developing at $0.7\left(2 e^{2} / h\right)$ can be observed in Fig. 2(c), bearing a strong resemblance to the "classic" 0.7 structure. The Kondo effect and the 0.7 structure appear

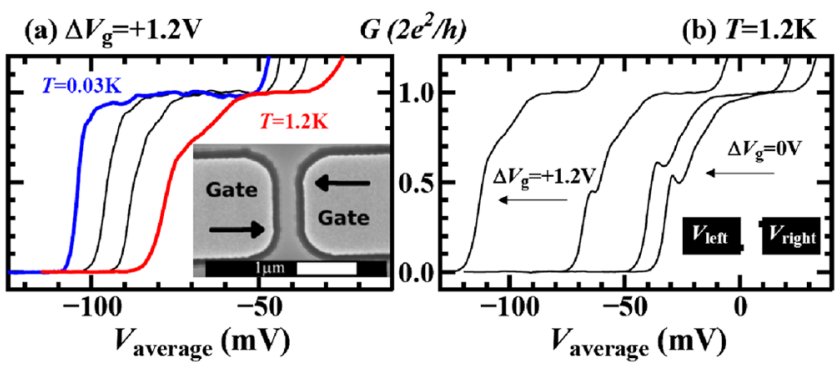

FIG. 1 (color online). (a) A classic 0.7 structure can be observed with $\Delta V_{g}=+1.2 \mathrm{~V}$ (traces offset laterally), defined as $\Delta V_{g}=V_{\text {left }}-V_{\text {right }}$, and $V_{\text {average }}=\left[V_{\text {left }}+V_{\text {right }}\right] / 2$. The inset shows a SEM image of our device; (b) the resonant peak disappears as $\Delta V_{g}$ is varied from 0 to $+1.2 \mathrm{~V}$ in $0.4 \mathrm{~V}$ steps from right to left traces. The inset shows a schematic diagram of the geometry of the narrow entrance and exit. 


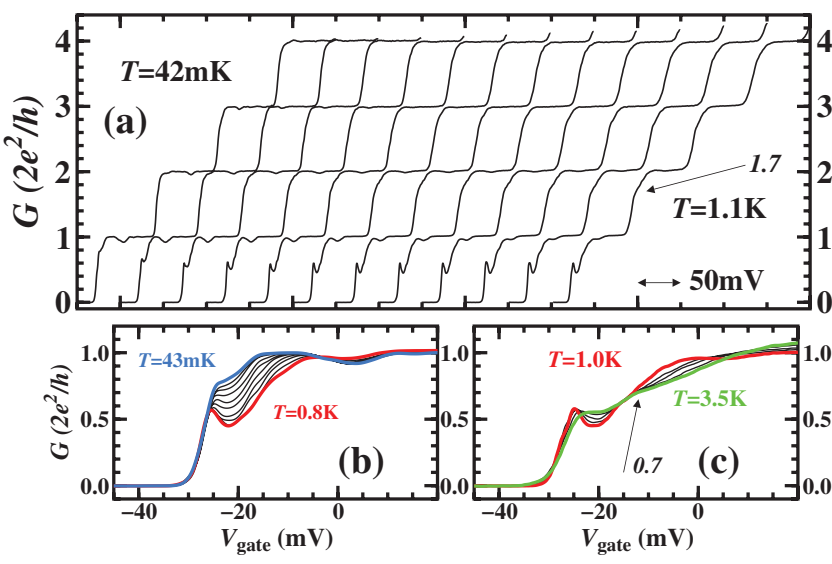

FIG. 2 (color). (a) Temperature is decreased from $1.1 \mathrm{~K}$ to $42 \mathrm{mK}$ (in $\sim 0.1 \mathrm{~K}$ increments; $\Delta V_{g}=0$; traces offset laterally). (b) The increase of $G$ as $T$ decreases is a hallmark of Kondo interactions $\left(\Delta V_{g}=0\right)$. (c) The $\mathrm{CB}$ peak smears out for $T \geq$ $1.0 \mathrm{~K}$ while a shoulder at $0.7\left(2 e^{2} / h\right)$ strengthens $\left(\Delta V_{g}=0\right.$; traces offset laterally).

to be two superimposed effects. Figure 2(c) is very similar to Fig. 2 of Ref. [22]. Looking back at Fig. 2(a), a shoulder at $1.7\left(2 e^{2} / h\right)$ also becomes more pronounced as temperature increases (indicated by an arrow). A very similar $0.7\left(2 e^{2} / h\right)$ structure and $1.7\left(2 e^{2} / h\right)$ feature were also observed at $T=1 \mathrm{~K}$ in Fig. 3 of Ref. [23], but without a Kondo-like effect.

From Fig. 2, we extracted $G$ versus $T$ at fixed $V_{\text {gate }}$. For $42 \mathrm{mK} \leq T<800 \mathrm{mK}$, we fitted the data with three models: the activation model $G_{A}=1-C_{A} e^{-T_{A} / T}$ [24], the quantum dot Kondo model $G_{K}=\left[1+\left(2^{1 / s}-1\right) \times\right.$ $\left.\left(T / T_{K}\right)^{2}\right]^{-s}$ [9], and the Kondo-like model $G_{K-L}=0.50+$ $0.50\left[1+\left(2^{1 / s}-1\right)\left(T / T_{K}\right)^{2}\right]^{-s}$ [4], where $G_{A}, G_{K}$, and $G_{K-L}$ are in units of $2 e^{2} / h$. We estimate our base electron temperature to be $\sim 42 \mathrm{mK}$. The Kondo-like model could not be fitted to our data. Figure 3 summarizes the fit results with the other two models, collapsed onto their universal curve. At high ratios $(>4)$ of $T_{A} / T$ and $T_{K} / T$, the data fit the quantum dot Kondo model better.

Figure 4 shows the effect of $V_{\text {sd }}$ on our device in both the $\Delta V_{g}=0$ and $\Delta V_{g} \neq 0$ regimes. In the $\Delta V_{g}=0$ regime, increasing $\left|V_{\text {sd }}\right|$ from 0 to $100 \mu \mathrm{V}$ at low $T$ has a dramatic effect: conductance is suppressed to reveal the CB peak normally visible only at higher $T$. One can directly compare the effect of $T$ in Fig. 2(b) and $V_{\text {sd }}$ in Fig. 4(a) (only $V_{\text {sd }}<0$ is shown). By contrast, in the $\Delta V_{g} \neq 0$ regime, increasing $\left|V_{\text {sd }}\right|$ up to $100 \mu \mathrm{V}$ has little effect [Fig. 4(b) shows $V_{\text {sd }}<0$ only]. In the proposed 1D Kondo-like model [4], the appearance of the so-called $0.85\left(2 e^{2} / h\right)$ plateau [24] is associated with the suppression of the Kondo-enhanced conductance from the $2 e^{2} / h$ plateau. In our devices, Figs. 4(c) and 4(d) show that the $0.85\left(2 e^{2} / h\right)$ plateau arises with increasing $V_{\text {sd }}$ in both the $\Delta V_{g}=0$ and $\Delta V_{g} \neq 0$ regimes (transition from the red trace to the green trace), well after the complete suppression of the Kondo
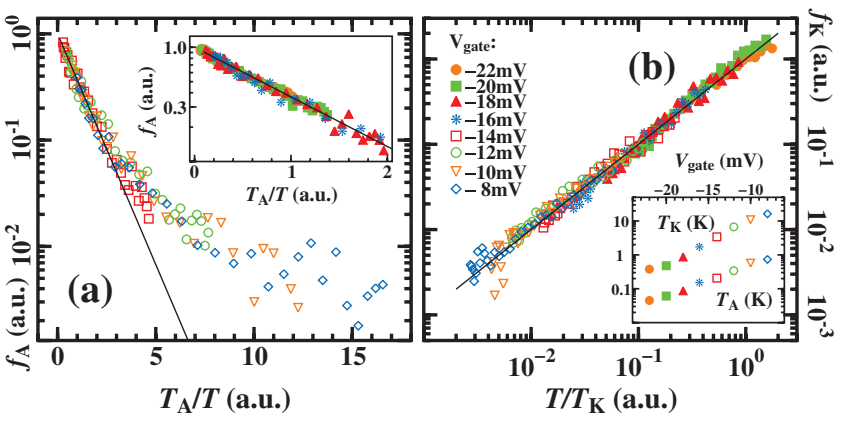

FIG. 3 (color). (a) Plot of $f_{A}=\left(1-G_{A}\right) / C_{A}$ versus $T_{A} / T$ for $-22 \mathrm{mV} \leq V_{\text {gate }} \leq-16 \mathrm{mV}$. The inset shows a detailed view of the range $0<T_{A} / T<2$ for $-14 \mathrm{mV} \leq V_{\text {gate }} \leq-8 \mathrm{mV}$. (b) Plot of $f_{K}=\sqrt{\left(G_{K}^{-1 / s}-1\right) /\left(2^{1 / s}-1\right)}$ versus $T / T_{K}(s=$ 0.11 ). The inset shows the extracted $T_{A}$ and $T_{K}$ ( $\left.\pm 5 \%\right)$, consistent with values found in previous studies [4,24]. In both (a) and (b), the black solid line shows the theoretical universal curve.

effect [transition from the blue trace to the red trace in Figs. 4(a) and 4(b)]. Moreover, Figs. 4(e) and 4(f) suggest the $\sim 0.3\left(2 e^{2} / h\right)$ and $0.85\left(2 e^{2} / h\right)$ plateaux appear regardless of whether $T<T_{K}$ or $T_{K}<T$. They are also often similarly observed in clean quantum wires [e.g., Figs. 1(d) and (e) in Ref. [4] and Fig. 1 in Ref. [25] ]. We believe their origin involves processes other than Kondo physics [20].
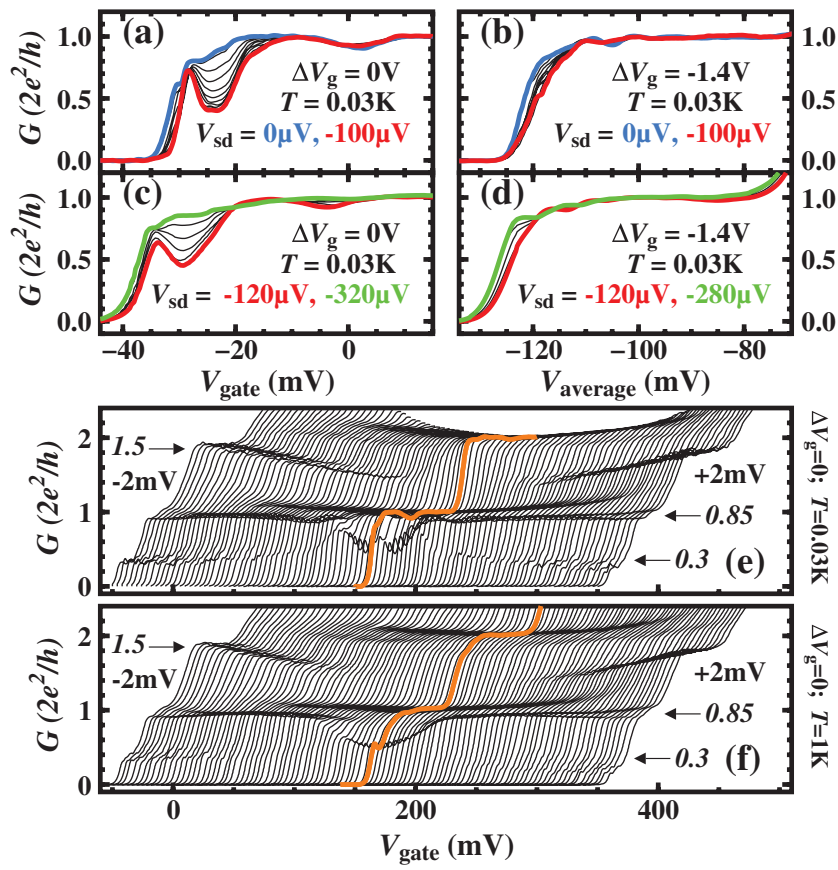

FIG. 4 (color). Low-temperature conductance with a sourcedrain bias when: (a), (c) $\Delta V_{g}=0 \mathrm{~V}$, and (b), (d) $\Delta V_{g}=$ $-1.4 \mathrm{~V}$. $\left|V_{\mathrm{sd}}\right|$ is increased from 0 to $100 \mu \mathrm{V}$ in (a) $-(\mathrm{b})$, and from 0.1 to $0.3 \mathrm{mV}$ in (c)-(d). High source-drain bias conductance for $\Delta V_{g}=0$ at: (e) $T=0.03 \mathrm{~K}$ and (f) $T=1 \mathrm{~K}$. From left to right (traces offset laterally), $V_{\mathrm{sd}}$ is increased from -2 to $+2 \mathrm{mV}$ in $40 \mu \mathrm{V}$ steps (orange traces show $V_{\mathrm{sd}}=0$ ). 
Figure 5 shows the effect of $V_{\text {sd }}$ on the zero-bias anomaly (ZBA) in the $\Delta V_{g}=0$ regime. There are three intervals of $V_{\text {gate }}$ values of interest shown in Fig. 5(a): "zone I" from pinch-off to the CB peak apex, "zone II" from the CB peak apex to the minimum of the Coulomb valley, and "zone III" from the minimum in the Coulomb valley up to the $2 e^{2} / h$ plateau. We further defined $G_{\max }$ as the maximum conductance achieved at $T=0.03 \mathrm{~K}, V_{\text {sd }}=$ $0 \mu \mathrm{V}$, and $B=0 \mathrm{~T} ; \Delta h_{\mathrm{ZBA}}$ is defined as $G_{\max }$ minus the conductance of the local minimum on the right-hand side of the ZBA. We do not use the ZBA width, as it is difficult to discern the ZBA from the expected bell shape of $d I / d V_{\text {sd }}$ when approaching a quantized plateau in zone III. Figures 5(b)-5(d) show that $G_{\max }$ increases monotonically with $V_{\text {gate }}$, so that Figs. 5(e) and 5(f) effectively plot $\Delta h_{\mathrm{ZBA}}$ as a function of $V_{\text {gate }}$. The $\Delta h_{\mathrm{ZBA}}$ minimum near $G_{\max } \sim 0.8\left(2 e^{2} / h\right)$ occurs at the apex of the CB peak, where first-order resonant tunneling through the bound state reaches a maximum and thus swamps the ZBA. In a quantum dot displaying the singlet Kondo effect, if the peak in the density of states associated with the single-particle state is fairly broad, a flat response in $G$ to very low $V_{\text {sd }}(<80 \mu \mathrm{V})$ can be observed on a CB peak [e.g. Fig. 4(d) in Ref. [8] ]. As shown by the black trace in Fig. 5(c), one can obtain such a flat response at precisely the CB peak apex. Interestingly, the ZBA clearly exists in both zones I and II at $B=0 \mathrm{~T}$, violating the odd-even parity rule for Kondo valleys [8] (two adjacent CB valleys cannot exhibit a singlet Kondo effect). Our results cannot
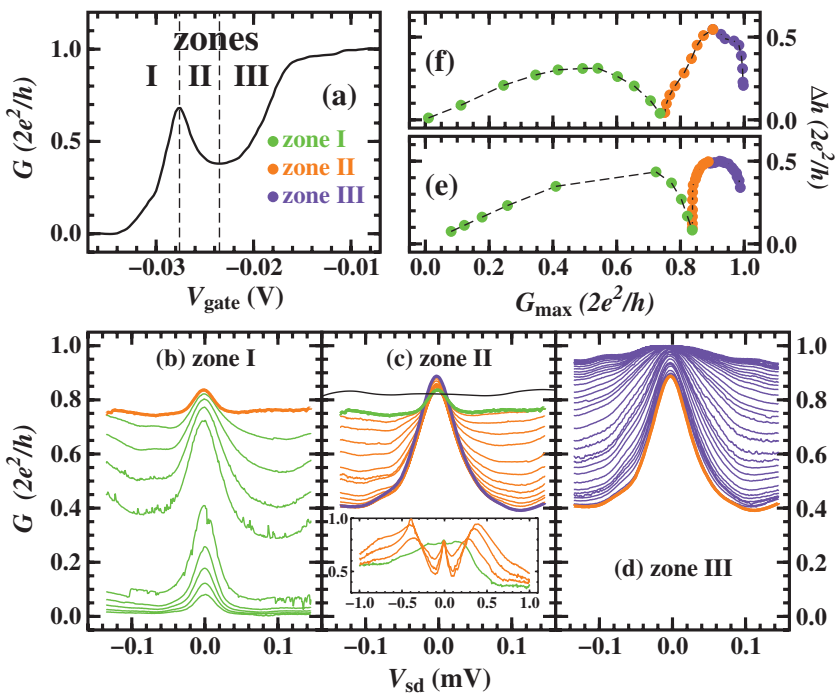

FIG. 5 (color). (a) "Map" of the $V_{\text {gate }}$ ranges defining zones I, II, and III $\left(\Delta V_{g}=0, V_{\text {sd }}=+100 \mu \mathrm{V}\right)$. Nonlinear differential conductance traces taken at fixed $V_{\text {gate }}$ at $T=0.03 \mathrm{~K}$ are shown for (b) zone I (green), (c) zone II (orange), and (d) zone III (purple). $V_{\text {gate }}$ is stepped by $0.3 \mathrm{mV}$ between traces. The black trace in (c) was taken at $T=0.04 \mathrm{~K}$ (see main text). The inset in (c) shows data at larger source-drain bias. $\Delta h_{\mathrm{ZBA}}$ (defined in main text) is shown: (e) after and (f) before illumination. Note how $\Delta h_{\mathrm{ZBA}}$ have a local minimum at the $\mathrm{CB}$ peak. be explained by adding in series the ZBA's of two independent systems (wire-wire, dot-wire) [26]. At intermediate values of $V_{\text {sd }}$ in zones I and II, a triple-peaked structure can be observed in $d I / d V_{\text {sd }}$ [see inset of Fig. 5(c)]. The central peak is the ZBA. We interpret the two satellite peaks to be resonant-tunneling peaks due to the alignment of the bound state with the Fermi level in the leads, allowing a direct measurement of the bound state's position in energy [27].

Figure 6(b) shows a similar triple-peaked structure in the $d I / d V_{\mathrm{sd}}$ of a clean quantum wire [also in Fig. 1(d) of Ref. [4], Fig. 3 of Ref. [24], and Fig. 2(b) of Ref. [28] ]. We hypothesize the two satellite peaks to the ZBA could be resonant-tunneling peaks due to an exchange-induced bound state (which we label $\epsilon_{1 \mathrm{D}}$ ) in the calculated double-barrier potential reported in Refs. [11-17]. Plotting $\Delta h_{\mathrm{ZBA}}$ against $G_{\max }$ or $V_{\text {gate }}$ for the ZBA in clean quantum wires, as shown in Figs. 6(c)-6(e), one often finds a local minimum where the 0.7 structure lies: firstorder tunneling through $\epsilon_{1 \mathrm{D}}$ reaches a maximum.

Figure 7(a) shows the effect of an in-plane magnetic field (parallel to the wire) on our device. The ZBA is spinsplit in Fig. 7(b): as $V_{\text {gate }}$ (and hence $T_{K}$ ) is decreased, we observe a transition from the regime $g^{*} \mu_{B} B<k_{B} T_{K}$ where spin-splitting cannot be resolved, to the regime $k_{B} T_{K}<$ $g^{*} \mu_{B} B$ where spin-splitting becomes visible (using the bulk GaAs Landé $g$ factor $g^{*}=0.44$, the Bohr magneton $\mu_{B}=58 \mu \mathrm{V} / \mathrm{T}$, and the $T_{K}$ values from Fig. 3). The variation in peak splitting is qualitatively consistent with a critical field $B_{c} \sim T_{K}$, as observed in quantum dots [29,30]. As with $T$ in Fig. 2(b) and $V_{\text {sd }}$ in Fig. 4(a), the Kondo enhancement of the conductance is rapidly suppressed with increasing field to reveal the CB peak. For $B \geq 1 \mathrm{~T}$, we suggest only spin-down electrons tunnel through the bound state, and thus the CB peak apex is pinned at $e^{2} / h$ while its right-hand side valley deepens with increasing field. The resonance on the $0.5\left(2 e^{2} / h\right)$ plateau is not mirrored on the spin-split $1.5\left(2 e^{2} / h\right)$ plateau:

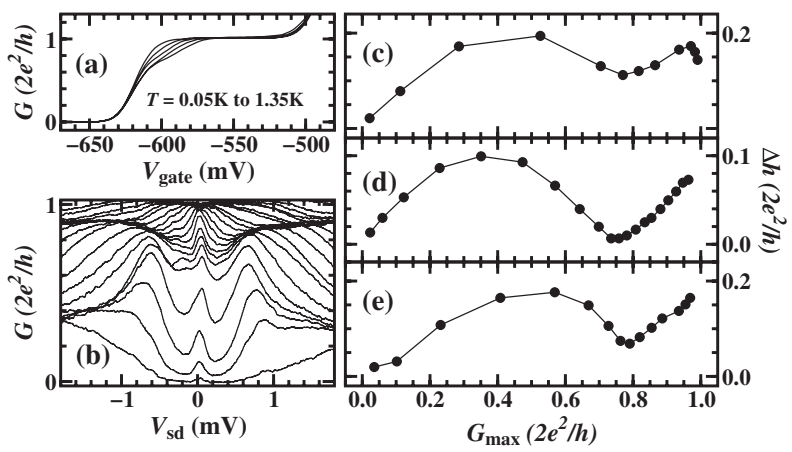

FIG. 6. (a) A classic 0.7 structure in a clean quantum wire. (b) Nonlinear differential conductance traces of the device shown in (a) taken for fixed $V_{\text {gate }}$ at $T=0.03 \mathrm{~K}$. Plots of $\Delta h_{\mathrm{ZBA}}$ are shown for: (c) data from panel (b), (d) data from Fig. 2(b) in Ref. [28], and (e) data from Fig. 1(d) in Ref. [4]. Note how all $\Delta h_{\mathrm{ZBA}}$ plots have a local minimum. 


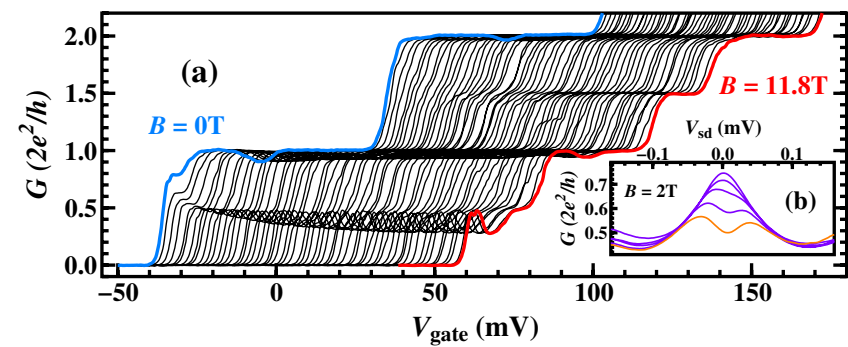

FIG. 7 (color online). (a) Magnetic field is increased from 0 to $11.8 \mathrm{~T}$ in $0.2 \mathrm{~T}$ steps from left to right (traces offset laterally, $T=0.04 \mathrm{~K}, V_{\text {sd }}=0$, and $\Delta V_{g}=0$ ). (b) Splitting of the ZBA for five decreasing values of $V_{\text {gate }}$ from zone III (purple, traces offset vertically) and zone II (orange, no offset).

it is not a length resonance [20]. Even in clean quantum wires that do not otherwise show any resonant peaks at zero or low magnetic fields, a resonance on the spin-split $e^{2} / h$ plateau often occurs (e.g., Figs. 1 in Refs. [31-33]). Unlike our open quantum dots, these resonances in clean quantum wires only become apparent at very large magnetic fields, on a fully developed spin-split $e^{2} / h$ plateau.

On a bound state in clean quantum wires. - Many of the characteristics of our open quantum dot devices are also shared by clean quantum wires, albeit in a much weaker form. These are (i) a well-defined conductance dip or an overall downward bow on the $2 e^{2} / h$ plateau at very low temperatures and $B=0 \mathrm{~T}$, (ii) a resonance on a fully developed spin-split $e^{2} / h$ plateau, deepening with increasing magnetic field, (iii) a triple-peaked structure in $d I / d V_{\text {sd }}$ at fixed $V_{\text {gate }}$ below the 0.7 structure, and (iv) a minimum near the 0.7 structure in plots of $\Delta h_{\mathrm{ZBA}}$ against $G_{\max }$ (or $\left.V_{\text {gate }}\right)$. This compels us to deduce that a bound or only partially extended state $\epsilon_{1 \mathrm{D}}$ can exist in clean quantum wires, near pinch-off. Whether the 0.7 structure is linked to this state $\epsilon_{1 \mathrm{D}}$ remains to be investigated.

On the 0.7 structure and Kondo physics. - In our open quantum dots, we were able to isolate phenomena associated with Kondo physics from that associated with " 0.7 structure" physics. For example, at high temperature in Fig. 2(c), there is an excess of conductance (for $V_{\text {gate }}>$ $-15 \mathrm{mV}$, past the shoulder) not fully accounted for by the Kondo model. Although we find that the Kondo effect is not responsible for all the 0.7 structure phenomenology, it does appear to be present in quantum wires (as embodied by the ZBA) at very low temperatures, magnetic fields, and source-drain biases. In light of the possible existence of a $\epsilon_{1 \mathrm{D}}$ state even in clean quantum wires, we thus propose that both the Kondo-like effect in 1D channels and the 0.7 structure are separate and distinct effects.

We thank A.C. Graham, V. Tripathi, J. Griffiths, D. Goldhaber-Gordon, Y. Meir, V. Puller, K. Matveev, K.-F. Berggren, N. Cooper, and K. J. Thomas for insightful discussions. For technical assistance, we thank K. Cooper, P. Flaxman, and A. Beckett. This work was supported by the EPSRC (UK) and the CCT (Cambridge).
*Corresponding author. fs228@cam.ac.uk

${ }^{\dagger}$ Present address: School of Physics, University of New South Wales, Sydney 2052, Australia.

[1] D. A. Wharam et al., J. Phys. C 21, L209 (1988).

[2] B. J. van Wees et al., Phys. Rev. Lett. 60, 848 (1988).

[3] K. J. Thomas et al., Phys. Rev. Lett. 77, 135 (1996).

[4] S. Cronenwett et al., Phys. Rev. Lett. 88, 226805 (2002).

[5] Y. Meir, K. Hirose, and N. S. Wingreen, Phys. Rev. Lett. 89, 196802 (2002).

[6] N.S. Wingreen and Y. Meir, Phys. Rev. B 49, 11040 (1994).

[7] D. Goldhaber-Gordon et al., Nature (London) 391, 156 (1998).

[8] S. M. Cronenwett, T. H. Osterkamp, and L.P. Kouwenhoven, Science 281, 540 (1998).

[9] W. G. van der Wiel et al., Science 289, 2105 (2000).

[10] M. Pustilnik and L. I. Glazman, J. Phys. Condens. Matter 16, R513 (2004).

[11] K. Hirose, Y. Meir, and N. S. Wingreen, Phys. Rev. Lett. 90, 026804 (2003).

[12] A. A. Starikov, I. I. Yakimenko, and K. F. Berggren, Phys. Rev. B 67, 235319 (2003).

[13] O. P. Sushkov, Phys. Rev. B 67, 195318 (2003).

[14] P. S. Cornaglia, C. A. Balseiro, and M. Avignon, Phys. Rev. B 71, 024432 (2005).

[15] A. Ramšak and J.H. Jefferson, Phys. Rev. B 71, 161311(R) (2005).

[16] T. Rejec and Y. Meir, Nature (London) 442, 900 (2006).

[17] P. Jaksch, I. Yakimenko, and K. F. Berggren, Phys. Rev. B 74, 235320 (2006).

[18] F. A. Maaø, I. V. Zozulenko, and E. H. Hauge, Phys. Rev. B 50, 17320 (1994).

[19] G. Kirczenow, Phys. Rev. B 39, 10452 (1989).

[20] F. Sfigakis et al. (to be published).

[21] M. J. Laughton, J. R. Barker, J. A. Nixon, and J. H. Davies, Phys. Rev. B 44, 1150 (1991).

[22] K. J. Thomas et al., Phys. Rev. B 61, R13365 (2000).

[23] D. J. Reilly et al., Phys. Rev. B 63, 121311(R) (2001).

[24] A. Kristensen et al., Phys. Rev. B 62, 10950 (2000).

[25] R. de Picciotto, L. N. Pfeiffer, K. W. Baldwin, and K. W. West, Phys. Rev. Lett. 92, 036805 (2004).

[26] See EPAPS Document No. E-PRLTAO-100-044801 for a PDF file which presents our arguments explaining why the exceptionally large ZBA we observe in our open quantum dot do not arise from the addition of the ZBA from two independent systems in series. For more information on EPAPS, see http://www.aip.org/pubservs/epaps.html.

[27] D. C. Ralph and R. A. Buhrman, Phys. Rev. Lett. 72, 3401 (1994).

[28] L. DiCarlo et al., Phys. Rev. Lett. 97, 036810 (2006).

[29] T. A. Costi, Phys. Rev. Lett. 85, 1504 (2000).

[30] S. Amasha, I. J. Gelfand, M. A. Kastner, and A. Kogan, Phys. Rev. B 72, 045308 (2005).

[31] K. J. Thomas et al., Philos. Mag. B 77, 1213 (1998).

[32] A.C. Graham et al., Phys. Rev. Lett. 91, 136404 (2003).

[33] A. C. Graham, D. L. Sawkey, M. Pepper, M. Y. Simmons, and D. A. Ritchie, Phys. Rev. B 75, 035331 (2007). 